\title{
An Acoustic Analysis for Infants Speech Syllables
}

\author{
Sisi Liu, Yasheng Jin, Hongzhi Yu
}

Key Lab of China's National Linguistic Information Technology, Northwest University for Nationalities, Lanzhou, 730030, China

\begin{abstract}
Phonological awareness, the ability to distinguish and make use of any phonetic unit contained in the word structure of colloquial language, involves two major aspects, manipulation of sounds, and detection of sounds. The former one can be subdivided into counting, separating, combining of syllables and substitution of phoneme, while the latter one mainly refers to distinguishing similarities and differences among the first syllables, the rhymes and tones. Based on syllables, this study analyzes the acquisition order reflected by the monosyllable and the multisyllables by tracking, recording and separating the syllables into more detailed phonemes, as well as induces the development of infants' phonological awareness from the aspects of pronunciation difficulty, spelling difficulty, related pronunciation development and the tone awareness through studies of the manipulation of sounds in the phonological awareness.
\end{abstract}

Keywords. infants; Complexity of syllables; Syllables; Phoneme; Formant

\section{Introduction}

Phonological awareness refers to the ability to distinguish and make use of any phonetic unit contained in the word structure of colloquial language ${ }^{[1]}$ (translated by the author). As for alphabetic writing, the research related to the phonological awareness involves syllable awareness, onset-rhyme awareness and phonemic awareness ${ }^{[2]}$ (translated by the author). Since Chinese pronunciation has special rules, the study of mandarin often related to research on the tone awareness. Phonological awareness involves two major aspects, manipulation of sounds, and detection of sounds. The former one can be subdivided into counting, separating, combining of syllables and substitution of phonemes, while the latter one mainly refer to distinguishing similarities and differences among the first syllables, the rhymes and tones. The close relationship between children's phonological awareness development and spelling and reading skills makes the phonological awareness draw more and more attention from both domestic and foreign scholars in recent years. Limited to time spend, the research in children's phonological awareness is still not comprehensive enough, particularly among the domestic studies. It is also a field that few scholars interested in when study the ideograph, such as Chinese. It has been found in most of the early study that pre-school children have a certain level of phonological awareness, with the syllable awareness appearing the first and the phoneme awareness appearing the last. Meanwhile, the study on the phonological awareness of children from Hong Kong and Taiwan is mainly focus on its 
relationship with the reading ability. However, the studies are limited on the phonological awareness of children speaking mandarin, particularly when they are in the early childhood. For the study of the phonological awareness of children speaking mandarin in China, it mainly concentrated in children in the primary schools, less being found among children in kindergarten or even earlier period. As a result, it is still unknown when the phonological awareness will occur and whether the syllable awareness will differentiate some phonological awareness.

\section{Research methods}

Voice samples in this study are taken from a two-year-old male infant whose parents documented his pronunciation in detail in their daily lives, using a recording pen with a sampling rate of $44 \mathrm{k}$, and the sample file is in wav format based on PCM coding. By recording the pronunciation development of this child, the research studies on the developmental process of his pronunciation and distinct characteristics in each stage. This article mainly focuses on the phonetic research of this child before three-years old. The samples are chosen from each single pronunciation of this infant, and are preliminarily divided into different groups according to the sense of hearing for further study. As for the splited samples, analyses are conducted form the aspect of syllables to testify that infants have phonological awareness in this period. When older than one year old, infants generally have the ability to distinguish between different syllables. Whether this syllable awareness is same to the syllable awareness in previous studies is also involved in this research.

\section{Monosyllable}

As one of the most unique monosyllabic language in the world, Chinese has special monosyllabic characters. Phoneme which can be divided into vowels and consonants is the smallest unit of syllables which consists of three major parts, initial consonants, compound vowels and tones. As all the consonants are acting as the initial consonants, and all vowels can act as compound vowels, vowels and compound vowels, consonants and initial consonants as well as syllables and phonemes have close relationships ${ }^{[3]}$ (translated by the author). During the maturing and learning in the infancy, monosyllable will first appear due to the short and easy pronunciation. While, as for learning monosyllable, infant will begin with the easiest single phoneme, then transit to double phonemes, three phonemes, four phonemes and even the phonemes of R-ending retroflexion.

\subsection{Single Phoneme}

Infants in three-month old learn to make several simple voices gradually, however, the voices are quite ambiguous and starting from single phoneme, not as standard as the adults' voices. The following graphic shows the two vowels which appear the first. (Figure 1)

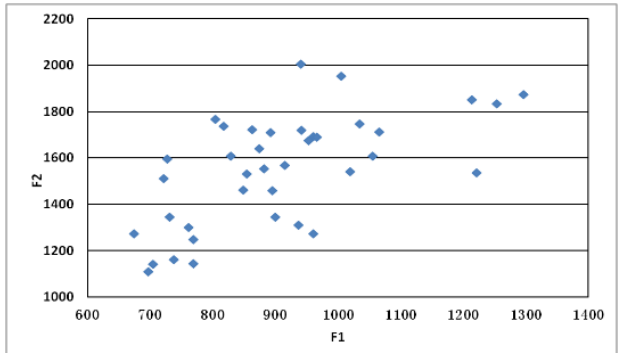

$/ \mathrm{a} /$

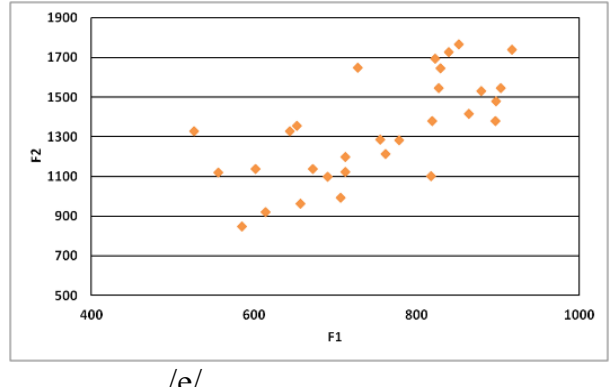

/e/

Figure 1. The distribution of vowel /a/ and /e/ in Figure 1 and Figure 2 
The left graphic demonstrates the distribution of /a/ in Figure 1 and Figure 2 for the threemonth-old infant. It is the pronunciation that appears the most in the voice samples during this period. In phonetics, / a / is an open vowel, with the tongue in the most natural position when pronounced. It can be found in the graphic that the Figure 1 of /a/ pronounced by the infant concentrated around $1000 \mathrm{~Hz}$, and that of Figure 2 is around $1500 \mathrm{~Hz}$. On another aspect, what the right graphic shows is that the situation the vowel /e/ which is also a pronunciation enjoys a high appearing frequency of 42 times in the voice samples during this period, being only next to the pronunciation of $/ \mathrm{a} / . / \mathrm{e} /$ is an half-open unrounded vowel which is not as indentified as the sound of /a/ when pronounce by infants, and still has some vague sound close to /a/. It can be found in the graphic that the Figure 1 of /e/ is around $750 \mathrm{~Hz}$ and that of Figure 2 is between $1200 \mathrm{~Hz}$ and $1600 \mathrm{~Hz}$.

Growing up to six-mouth old, infants acquire more types of vowels, such as /i/ and /u/. Though the new vowels are not in large mount, they can be clearly indentified. During the research, the formants of / $\mathrm{i} /$ and / $\mathrm{u} /$ have been extracted, and the range of the first and the second formant have been summed up and compared in the following table (table 1).

Table 1. The Comparison of formants of / i / and / $\mathrm{u} /$ for six-month

\begin{tabular}{|l|l|l|l|l|}
\hline & Infant F1 & Adult F1 & Infant F2 & Adult F2 \\
\hline$/ \mathrm{i} /$ & $560-600 \mathrm{~Hz}$ & $250 \mathrm{~Hz}$ & $1350-1600 \mathrm{~Hz}$ & Above $2000 \mathrm{~Hz}$ \\
\hline$/ \mathrm{u} /$ & $490-510 \mathrm{~Hz}$ & $300 \mathrm{~Hz}$ & $1050-1300 \mathrm{~Hz}$ & $600 \mathrm{~Hz}$ \\
\hline
\end{tabular}

Due to the high limits of vocal organs' development, the first pronunciation of the infant is the pronunciation produced when the tongue is in the most natural state. Then, with the development of pronunciation acquisition, there will be more and more changes in tongue positions, but infants cannot yet pronounce accurate voices at once. Figure 1 is inversely proportional to the height of tongue position, and the higher of it, the tongue states lower. While, Figure 2 is proportional to the back and forth position of tongue, and the higher of it, the tongue moves more front. Thus, the vowels $/ \mathrm{i} / \mathrm{and} / \mathrm{u} /$ pronounced by the infant during this period are different from those by adults. It is specifically reflected in the following aspects: tongue position of the vowel $/ \mathrm{u} /$ is more front and tongue position of the vowel / $\mathrm{i}$ / is more back. Although they are greatly different from adults, children of this period has been basically learned to pronounce the single phoneme.

\subsection{Double Phonemes}

After the baby was born for three months, there will appear the sliding flow of sounds which refers to the uninterrupted flow of syllables, including the sliding flow of vowels, the vowel flow containing consonants, the sliding flow of single phoneme and double phonemes as well as the sliding flow of mix sounds ${ }^{[4]}$ (translated by the author). As for children's language development, the learning of double phonemes mainly focuses on the double vowels and consonants plus single vowels. In the sixth month or even earlier, infants can pronounce the double phonemes such as mama and baba through reduplicated sounds, and the concept of syllables appears in the twelfth month to be clearly indentified the differences between the sliding flows of double vowels and the sliding flows due to mixed factors. The following analysis will be conducted with the examples of the sound /a-u/ and the compound vowels /au/, being clearly demonstrated in the 3-D sonogram below. (Figure 2) 


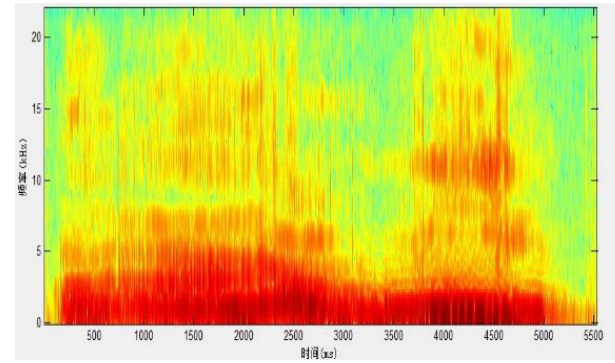

Figure 2. the sonogram of /a-u/

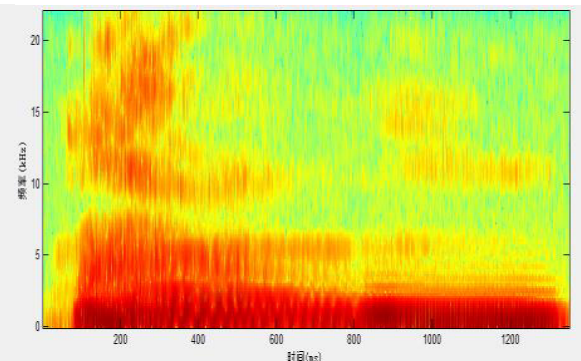

the sonogram of /au/

In the above graphic, the left one is the 3-D sonogram of / au / in which it can clearly be find the differences between the current two pronunciations and these pronunciations learned before. It is not a transitional process, but can be clearly seen in the sonogram of /au/ that it is a continuous pronunciation without being divided. From the differences between two parts of the above graphics, we can see that there appear syllables in the pronunciation of infants during this period, which also reflects that infants at about one year old have already form the preliminary concept of syllables, and can performance these differences by listening and pronouncing sounds.

\subsection{Three-phonemes}

As the acquisition of double phonemes, three-phonemes and even four-phonemes are evolved by duplicated words, the occurrence of these words make preparation for the development of children's pronunciation. Through continuous exercises, children will eventually master this particular pronunciation. Three-phoneme syllables can be divided into two major types. One is the general three-phoneme syllables, and the other one is the three-phoneme with R-ending retroflexion. The common three-phoneme, appearing in duplicated words, is concentrated in the combinations of open vowels with different bilabial, apical consonants and nasal sounds, such as /dai/, /dei/, /nai/ and /mao/, and can be splited by listening. On the other side, the three-phoneme with R-ending retroflexion focuses on adding an $\mathrm{R}$ retroflexion at the end of nasal sounds.

The maturing of articulator enables the infants' pronunciation being more flexible and various in types. During analyzing the splitted voices of 18-mounth-old infants, it has been found that Rend retroflexion appears as different from the past pronunciation. The pronunciation of the sound with R-end retroflexion can be seen as a two-phrase process. In the first phrase, the root of the tongue retracts and lays upward close to the soft palate, meanwhile, the soft palate backwards near to the back inner side of vocal track to block the nasal passage, forming two vocal tracks with the front one larger than the back one and having a little inferior track behind the tongue. The mouth opens round; similar to what it is when the low central vowel /a/ is pronounced. Then, in the second phrase, the tip of the tongue goes back and upward, to form a larger track behind the tongue with the back of the vocal track being smooth and longer. From the perspective of acoustics, the retroflexion in the R-ending pronunciations is related to Figure 2 and Figure 3. The tip of the tongue upturns higher, the heavier the retroflexion will be, and the lower of Figure 3 which will be much closer to Figure 2.

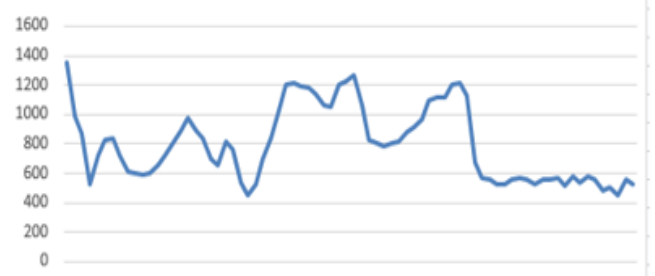

Figure 3. the difference curve of Figure 2 and Figure 3 for the R-ending 
The whole pronunciation process of /guangr/ can be seen from picture3, and its characters of formant is as same as other R-ending. It is obviously that the general difference value which is between Figure 2 and Figure 3 is above 600, and it reduced at the end, which is about 500.

Although the types of R-ending retroflexion are not various like in other periods, they can still reflect the fact that the tongue of the infant moves more and more flexible, and that the physical development of the infant, particularly the development of the tongue gets more and more mature.

\section{Multi-syllables}

The first multi-syllables that an infant learns is /mama/, which is not only in Chinese, but also in various languages. With growing up, the vocal organs continue to mature and the infant can pronounce two or even three syllables.

\subsection{Double Syllables}

During the six-month study, it has been found that infants can pronounce two syllables /mama/ and $/ \mathrm{baba} /$, and the pronunciation of /dada/ and /babu/ appears in their ninth month. The first sound that infants can pronounce is $/ \mathrm{mama} /$, regardless of which country are they in $^{[5]}$ (translated by the author). The other double syllables are constituted based on the bilabial consonant $/ \mathrm{b} /$ and the apical consonant $/ \mathrm{d} /$. This phenomenon also displays that the major vocal organs of infants in this period are lips and the tongue and the use of the tongue starts from its tip. When grow to twelvemonth old, infants can pronounce /babi/, /babu/, /dadi/ and /dadu/ which shows no breakthroughs by also relating to new types of vowel-consonant combinations and mainly focusing on the bilabial and apical pronunciations as before. However, the combinations of bilabial and apical pronunciations with syllables with /i/ or / $/$ / as the final vowel or a final vowel beginning with /i/ or $/ \mathrm{u} /$ demonstrate the development of flexibility of lips and the tongue. The types of double-syllables in pronunciation of infants during this period are diversified and numerous, as in the following table (Table 2):

Table 2. Types of double-syllables for eighteen-month-old infants

\begin{tabular}{|l|l|}
\hline Consonants & Double-syllables \\
\hline$/ \mathrm{b} /$ & baba, babu, babi, bibi, bobo, biubiu \\
\hline$/ \mathrm{d} /$ & dada, dadu, didi \\
\hline$/ \mathrm{g} /$ & gaga, gege, gougou \\
\hline$/ \mathrm{m} /$ & mimi, meimei, miemie \\
\hline$/ \mathrm{n} /$ & nana, nainai \\
\hline$/ \mathrm{j} /$ & jiejie \\
\hline$/ \mathrm{zh} /$ & zhizhi \\
\hline
\end{tabular}

The above table demonstrates the major types of double-syllables in expression of infants during this period. The types of consonants involves the bilabial consonant, the apical consonant, the radical consonant, the palatal consonant and the nasal consonant, and they prove that infants gain significant process in acquiring consonants during one and a half years old, and most of them are pronounced as duplicated words. In spite of this, problems still exist in the learning of consonants for infants, which is that most of the pronunciation learning during this period is for unaspirated consonants, while the pronunciation of aspirated consonants still need more practice, and this shall be pay more attention to in the future studies. 


\subsection{Three-syllables}

The three-syllables appears when infants grow up to twelve-month old, and they mainly include /douyaya/, /laoyeye/, /daguanrguanr/, /xiaoguanrguanr/, /xiaomaomi/ and /damaomi/, as in the following graphic (Figure 4):

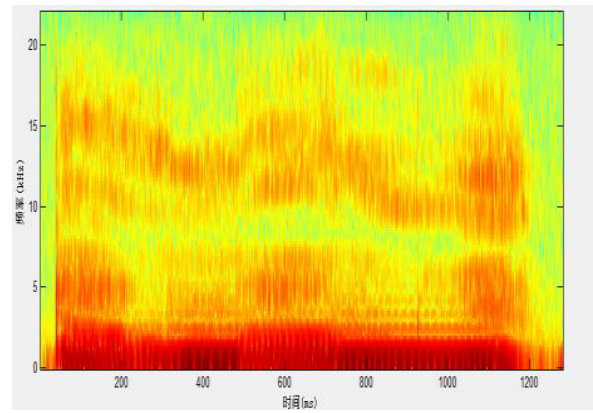

Figure 4. The sonogram of /damaomi/

The above graphic shows the sonogram of the three-syllable word /damaomi/, from which we can clearly identify the distribution of these three syllables. The rushed straight in the position of around $40 \mathrm{~ms}$ characterized the consonant $/ \mathrm{d} /$. Around $300 \mathrm{~ms}$ is the dividing line of the first and second syllable, and around $750 \mathrm{~ms}$ is the dividing line of the second and the third syllable.

The surging of multi-syllables surge in this period reflects that infants begin to speak more words and phrases, and most of the three-syllables are in the format of $\mathrm{ABB}$ due to the cognitive changes that infants experienced by understanding how to distinguish objects by their characteristics, such as the differences between big cat and kitty, the different size for jars and the differences between grandfather and the male elder.

\section{Conclusions}

The phonological awareness of infants begin in the earliest phase of pronunciation acquisition, which contains the awareness of the monophonic voice, the phoneme, the units containing in a syllable and the phoneme. This paper focuses on the tracking research of the awareness of the monophonic voice, the syllables as well as part of the units containing in a syllable.

(1) The influence of difficulty of pronunciation on the awareness of the monophonic voice. This awareness in infants' pronunciation acquisition is influenced by the difficulty of pronunciation, and the development and flexibility of vocal organs limits the different process for pronouncing different monophonic voice, reflecting in the acquisition order for vowels and consonants respectively. In the aspect of vowels, usually, the tongue tie extends to or near the tip of the tongue for newborns. As it will cause limits to the movement of the tongue, the earliest voice that the infant can make is when the tongue in the most natural position. Along with growing up, the tongue tie will gradually back to the root of the tongue, which led the tongue being more and more flexible, and the labium frenum and the buccal frenum further develop. The vowel generally develops from the lingual surface to the tip of the tongue, from non-rolling to rolling, from unrounded lips to rounded lips, from the inferiority to superiority and from the front to the back. Then consonants of infants presents by jumping from the back to the front getting closer to the middle part. Most of the earliest consonants are from the back of vocal organs such the uvula and the pharynx. With the flexible movement of the lips, the bilabial consonants appear, and then follow by the radical consonants, labiodentals and the lingual consonants.

(2) Difficulty in spelling impacts on the awareness of syllables. The awareness of syllables in infants' pronunciation acquisition can be divided into different phases due to the different difficulty in spelling words. From the monophonic perspective, the single phoneme develops into multi-phonemes and the infants eventually learned how to spell the monophony with three 
syllables as well as the R-ending retroflexion. The double-syllables and the three-syllables which appear the earliest are consisted by simple vowels and consonants. With the maturing of pronunciation, the spelling syllables getting more and more difficult.

(3) The relevance of voice development. The occurrence of vowels and consonants are attendant. The pronunciation acquisition of infants is not totally separated for vowels and consonants and it is an interleaved process by conducting mutual promotion and influence. Besides, in the same phase, their pronunciation may be limited to the same difficulty. When most of the vowels are low central vowels, the consonant are around the back of vocal organs. By the same token, the apical vowels are along with the bilabial and apical consonants; while when the back vowel with the root of the tongue uplift appears, there follows the radical consonant. Furthermore, the retroflex vowel demonstrates that the tongue moves more flexibly.

(4) The reflection of the tone awareness. Tone awareness is different from the monosyllable to the multi-syllable with the former being easier to pronounce. Therefore, the tone awareness appears in the earlier stage of monosyllable acquisition. Infants in six-month old can performance different tones of low central vowel /a/, and will gradually improve the tone acquisition of monosyllables. Meanwhile, due to the difficulty of pronunciations of multi-syllables, the acquisition for this part appears a bit late for infants, and some of the multi-syllables are pronounced inaccurate.

\section{Acknowledgement}

This research is supported by Key Laboratory of National language intelligent processing, Gansu Province (Northwest University for Nationalities).

\section{References}

1. Jiang Tao, Peng Danling. Phonological Awareness: Overview of the Theoretical Perspectives and Related Researches [J]. Journal of Developments in Psychology, 1996(3): 1-6.

2. Tang Shan, Wu Xinchun. The Early Development of Phonological Awareness for Chinese Children [J]. Psychological Science, 2009(2): 312-315.

3. $\mathrm{Xu}$ Baoliang, The Research on the Development of Phonological Awareness for Chinese Children and Its Enlightenment [J]. The Journal of Investigation and Research, 2005(12): 1920.

4. Li Yuming, The Development of Children's Languages [M]. Hubei: Huazhong Normal University Press.

5. Liao Zhilin, Studies on the Earliest Vocal Language based on the First Sound /mama/ Made by Infants [J]. The Academic Journal for San Menxia Vocational and Technical College, 2007(2): 49-55. 